\title{
HEMODIAFILTRATION BENEFICIALLY AFFECTS QT INTERVAL DURATION AND DISPERSION COMPARED TO HEMODIALYSIS
}

Kitti Barta $\mathrm{MD}^{1}$, Árpád Czifra $\mathrm{MD}^{1}$, Csaba Kun $\mathrm{MD}^{2}$, Alida Páll MD ${ }^{1}$, Julianna Kulcsár $\mathrm{MD}^{1}$, György Paragh MD, $\mathrm{DSc}^{1}$, István Lörincz $\mathrm{MD}, \mathrm{PhD}^{1}$, Tamás János Padra $\mathrm{PhD}^{1}$, Anupam Agarwal ${ }^{3}$ MD, Abolfazl Zarjou ${ }^{3}$ MD, József Balla ${ }^{1}$ MD, DSc, Zoltán Szabó MD, $\mathrm{PhD}^{1}$

${ }^{1}$ Department of Medicine, Division of Nephrology, Medical and Health Science Centre, University of Debrecen, Hungary

${ }^{2}$ Department of Cardiology, Medical and Health Science Centre, University of Debrecen, Hungary

${ }^{3}$ Department of Medicine, Nephrology Research and Training Centre and Centre for Free Radical Biology, University of Alabama at Birmingham, Birmingham, Alabama

Corresponding author: $\quad$ Zoltán Szabó, $\mathrm{MD}, \mathrm{PhD}$

Division of Nephrology, Department of Medicine

Medical and Health Science Centre, University of Debrecen

Pf. 19.

Nagyerdei krt. 98.

4012 Debrecen, Hungary

Phone: +36 302589494

E-mail: szaboz.med@gmail.com

Short title: Effect of hemodiafiltration on QT interval

Disclosure Statement: No conflicts of interest to disclose

Word count: 2549

Key words: hemodiafiltration, hemodialysis, ventricular arrhythmias, QT interval, QT dispersion 


\begin{abstract}
Background/Aims: The prolongation of the QT interval and dispersion could predict ventricular arrhythmias. It is not yet established whether there is a difference between the effects of hemodialysis and hemodiafiltration on QT interval duration and dispersion.

Methods: Data of thirty patients was investigated while they were receiving hemodiafiltration over a period of three months then the same group of patients was evaluated during treatment with conventional hemodialysis for at least another three months. Ionic parameters, surface electrocardiograms (ECG) were analyzed five times during each session, and 2D, M-mode echocardiography, Holter ECGs were performed to acquire additional information.
\end{abstract}

Results: QT interval duration (QTmax) and dispersion (QTd) showed a significant increase during hemodialysis, but not during hemodiafiltration. QTmax was $388.66 \pm 31.81 \mathrm{msec}$ at the beginning of hemodialysis, and increased to $400.66 \pm 39.12 \mathrm{msec}$ even at the $30^{\text {th }}$ minute ( $\mathrm{p}<0.05$ ). QTd was found to be $31.33 \pm 10.08 \mathrm{msec}$ before the commencement of hemodialysis with the largest prolongation being seen at the $240^{\text {th }}$ minute $(51.33 \pm 14.56 \mathrm{msec}, \mathrm{p}<0.05)$. The occurrence of ventricular premature beats was significantly higher during hemodialysis $(p=0.018)$. The left atrial diameter significantly decreased at the end of hemodiafiltration (at the beginning $45.1 \pm 5.25 \mathrm{~mm}$, at the end $40.77 \pm 5.76 \mathrm{~mm}$; $\mathrm{p}<0.05$ ).

Conclusion: Our results suggest a beneficial effect of hemodiafiltration on the studied electrocardiographic parameters compared to hemodialysis. The larger decrease in the left atrial diameter suggests a more efficient intracardiac volume decreasing potential of hemodiafiltration. 


\section{Introduction}

QT interval represents the electrical repolarization of the ventricular myocardium. It is dependent on the ventricular rate (the faster the heart rate the shorter it is) and can improve the detection of patients at increased risk of life threatening ventricular dysrhythmias. The QT interval duration varies between leads on the standard surface electrocardiogram. These interlead differences called QT dispersion, which is an index of the spatial dispersion of the ventricular recovery times, and can distinguish between myocardium that is homogeneous from myocardium that displays increased prolongation of repolarization [1-5] Several studies have highlighted the increased susceptibility of developing ventricular arrhythmias during hemodialysis. Cardiovascular mortality accounts for $40 \%$ of all deaths of patients suffering from kidney failure, where sudden cardiac death occurs in approximately $60 \%$ of patients undergoing hemodialysis[6,7]. Whereas conventional hemodialysis eliminates mostly small molecular weight uremic toxins by diffusion, hemodiafiltration has the advantage of eliminating higher molecular weight toxic polypeptides by convective transport. In comparison to hemodialysis, the quality of life is superior and mortality rates are lower in the case of hemodiafiltration[8-10]. Moreover despite the huge changes with regard to volume status the quality of life is improved and mortality rates are lower in the case of hemodiafiltration. However, the effects of hemodiafiltration on ventricular arrhythmia tendency have not yet been elucidated. Also, it is not clear to what extent the changes in ventricular arrhythmia susceptibility affect the mortality rate, compared to conventional hemodialysis. Therefore, the question is whether electrocardiographic markers, reflecting ventricular repolarization, differ with renal replacement modalities.

\section{Patients and methods}

Thirty patients, with end stage renal failure were enrolled during a single center study from our extracorporeal center (18 males, 12 females, mean age 60.57 \pm 13.62 years, range 23-85 years). Firstly, we collected and analyzed the data of the patients while they were under hemodiafiltration for three months, then the same patients were treated with conventional hemodialysis for at least another three months. This was followed, at the time of the regular hemodialysis, by another data collection. All diseases which can affect pulse generation and conduction of the heart were exclusion criteria. Thus, patients with amyloidosis, sarcoidosis, carcinoid, hemochromatosis, diabetes mellitus or Parkinson's disease were not involved in our 
investigations. QT interval may be affected by genetic causes (long QT syndrome, short QT syndrome), moreover the prolongation of the QT interval may be the consequence of drug interaction (e.g. haloperidol, methadone, amiodarone, sotalol, selective serotonine reuptake inhibitors, macrolide antibiotics, and antifungal agents). Likewise, hypothyroidism and altered calcium metabolism may affect this electrocardiographic parameter. Patients on these medications and/or suffering from such endocrine disorders were also not involved in the study. None of our patients suffered from inherited long or short QT syndrome. Patients with atrial fibrillation were also excluded. Patients suffering from end stage kidney disease (Stage 5) participating in regular hemodialysis program in our center, and were willing to give their informed consent to take part in the investigations were included. Chronic renal failure was caused by the following: chronic glomerulonephritis $(n=5)$, hypertensive and vascular nephropathy $(n=12)$, chronic pyelonephritis $(n=1)$, polycystic kidney disease $(n=2)$, analgesic nephropathy $(n=3)$, renal agenesis $(n=1)$, lupus nephritis $(n=2)$, and vasculitis $(n=4)$. Clinical data of the study population are shown in Table 1. All patients gave informed consent to participate in the study and the Institutional Ethics Committee on Human Research approved the study protocol. Hemodialysis and hemodiafiltration were performed three times a week in 4-hour long sessions with Fresenius $4008 \mathrm{~S}$ and H machines (Fresenius Medical Care, Bad Homburg, Germany), and with Fx60 and Fx80 high-flux polysulfone dialyzers (Fresenius). During hemodiafiltration $15.16 \pm 5$ liters of ultrafiltrate was removed. All of our patients were treated with post-dilution method during hemodiafiltration sessions. The replacement solution was manufactured on-line from ultrapure water and consisted of $138 \mathrm{mmol} / \mathrm{L}$ sodium, 2 or 3 $\mathrm{mmol} / \mathrm{L}$ potassium (in 13 cases $2 \mathrm{mmol} / \mathrm{l}$ in 17 cases $3 \mathrm{mmol} / \mathrm{l}$ ), $1.5 \mathrm{mmol} / \mathrm{L}$ calcium, 0.5 $\mathrm{mmol} / \mathrm{L}$ magnesium, and $1 \mathrm{~g} / \mathrm{L}$ glucose. The blood flow was $338 \pm 11.6 \mathrm{ml} / \mathrm{min}$ and did not differ significantly during the respective procedures $(\mathrm{p}<0.05)$. The bicarbonate dialysis solution contained the same concentrations. During the sessions no drugs other than isotonic sodium chloride and sodium heparin solutions were used. The serum ion levels were measured four times during the sessions and 2 hours afterwards. The arterial blood pressure was monitored non-invasively. The conventional 12-lead ECGs were performed five times in each case: at the beginning of the treatment, and in the $15^{\text {th }}, 30^{\text {th }}$ and the $240^{\text {th }}$ minutes, and two hours after the end of the sessions. Electrocardiograms were recorded at $25 \mathrm{~mm} / \mathrm{sec}$ recording speed (Hewlett Packard Page Writer 200i) while the patients were in the supine position. Copies of the recordings were made with a magnifying factor of 3 ; in every lead three QT intervals were measured. In order to eliminate the inter-observer variability the values were calculated with calipers by one examiner in a blinded fashion. The end of the T- 
wave was determined as the beginning of the TP interval; in the case of the U-waves, the deepest point between the $\mathrm{T}$ - and $\mathrm{U}$-waves marked the end of the interval. Three consecutive sections were defined, then their average was calculated and the resulting value used as the QT value in the given lead. In the statistical analysis, the longest QT value of the 12 leads was used as the QT interval (QTmax), and the QT dispersion (QTd) was determined as the difference between the longest and shortest QT interval. The QT interval and QTd were corrected to heart rate (QTmax c, QTd c) according to Bazett's formula: QTmax c $=\mathrm{QT} / \sqrt{\mathrm{RR}}$ $(\mathrm{msec})$. Before and after the renal replacement therapy transthoracic echocardiography (Mmode, 2D) were performed with pulsed and continuous wave Doppler technique (Philips ATL HDI 5000 imaging system with a $3.5 \mathrm{MHz}$ transducer was used). During the examinations left atrial cross diameter and left ventricular ejection fraction were determined (Simpson's formula). Left ventricular mass index was calculated with the equation suggested by Devereux and Reichek (\{1.04x [(end-diastolic diameter of the left ventricle + interventricular septum thickness + the posterior wall thickness of the left ventricle) ${ }^{3}$-end-diastolic diameter of the left ventricle $\left.\left.{ }^{3}\right]-14\right\}$ /height). Holter-ECGs (GE Medical SEER Light) were performed to assess ventricular arrhythmias, and were started before the therapy and ended 24 hours afterwards thus, both intra- and interdialytic periods were monitored. The number of ventricular premature beats was compared to the total number of beats and the resulting modulus was used to eliminate the variations arising from the short differences between the duration of the examinations.

\section{Statistical analysis}

The statistical analysis was carried out with the help of SAS 8.2 for Windows.

The variations of the investigated parameters over time and the differences between the two modalities were investigated by using ANOVA. The correlations between the parameters were analyzed by using the Pearson test when the distribution was normal and by Spearman's rank test in the case of an abnormal distribution. Throughout the analysis the $\mathrm{p}<0.05$ probability level was considered to be statistically significant.

\section{Results}

All electrocardiographic parameters showed an increase during hemodialysis $(\mathrm{p}<0.05)$, but no significant changes were observed during hemodiafiltration. Even all the starting ECG parameters were significantly longer in case of hemodiafiltration compared to hemodialysis 
(QTmax p<0.0001, QTc p<0.0001, QTd p=0.0034, QTd c p=0.003). Nevertheless, all of the studied electrocardiographic markers were found to be within the physiologic range at the beginning of the sessions. Neither QTmax and the QTd nor QTmax c and QTd c showed significant changes during the first 30 minutes of the hemodialysis, but from the $30^{\text {th }}$ minute prolongation was observed in all parameters $(\mathrm{p}<0.05)$. The average value of QTmax was $388.66 \pm 31.81$ milliseconds (msec) at the beginning of hemodialysis, and increased to $400.66 \pm 39.12 \mathrm{msec}$ by the $30^{\text {th }}$ minute $(\mathrm{p}<0.05)$ and reaching its maximum value by the $240^{\text {th }}$ minute of the hemodialysis $(418.67 \pm 46.07 \mathrm{msec}, \mathrm{p}<0.05)$, QTmax remained prolonged, compared to the base value $(391.33 \pm 43.2 \mathrm{msec})(\mathrm{p}<0.05) 2$ hours following the hemodialysis. The mean value of the QTd was found to be $31.33 \pm 10.08 \mathrm{msec}$ before the commencement of the hemodialysis with the largest prolongation being seen at the $240^{\text {th }}$ minute $(51.33 \pm 14.56$ msec, $\mathrm{p}<0.05$ ); this value, compared to the base value, was still persistently prolonged at $34.66 \pm 12.79 \mathrm{msec}$ two hours following the treatment. QTmax c had lengthened from the base value of $435.4 \pm 28.42 \mathrm{msec}$ to $454.93 \pm 32.76 \mathrm{msec}$ at thirty minutes, and this value kept on increasing. Even after these values started decreasing, we still found that the prolongation was significant 2 hours after the treatment $(453.26 \pm 28.00 \mathrm{msec}, \mathrm{p}<0.05)$. QTd c rose from $34.96 \pm 10.95 \mathrm{msec}$ to $43.46 \pm 13.28 \mathrm{msec}$ in the first half hour of the hemodialysis $(\mathrm{p}<0.05)$ and reached its maximum value by the $240^{\text {th }}$ minute $(58.27 \pm 15.54 \mathrm{msec}, \mathrm{p}<0.05)$. In contrast, the parameters of the QT interval did not change significantly during the hemodiafiltration (Figures 1. and 2.). The total calcium and ionized calcium showed an increase $(p<0.05)$, while serum potassium, magnesium and phosphate concentrations decreased significantly during both renal replacement modalities (HDF $\mathrm{p}<0.05$, HD: $\mathrm{p}<0.05$ ). However, we did not find significant differences between hemodialysis and hemodiafiltration regarding these parameters (Table 2.). During hemodiafiltration sodium levels did not change significantly, however, in the case of hemodialysis a decrease was observed in the $15^{\text {th }}$ and $30^{\text {th }}$ minutes $(\mathrm{p}<0.05)$ compared to the baseline levels (Figure 3.). During hemodiafiltration, a negative correlation was seen between both the total calcium and ionized calcium levels, and the QTmaxc; while in the case of hemodialysis the serum sodium concentration and the QTmaxc showed a positive correlation (Table 3.). Changes in ventricular rate were characterized by the RR cycle length. During hemodiafiltration the RR length was increased $(852 \pm 104.3 \mathrm{msec}$, $\mathrm{p}<0.05$ ) at the $30^{\text {th }}$ minute, indicating the provisional decrease of the heart frequency, and then it decreased. In the case of hemodialysis, the RR cycle length was observed to decrease $(800.6 \pm 96.2$ vs. $746.6 \pm 125.5 \mathrm{msec}, \mathrm{p}<0.05) 2$ hours after treatment (Figure 4.). Regarding blood pressure in both treatment modalities a rapid decrease occurred after the start of the 
sessions, which compared to baseline values, reached a significant level by the $15^{\text {th }}$ minute. The systolic blood pressure did not change significantly after this, however, the diastolic values showed an increase after the treatment. During hemodiafiltration the systolic and diastolic values were higher even at the initial stages of the sessions. (Figures $\mathbf{5 / a}$. and $\mathbf{5} / \mathbf{b}$.) At the beginning there were no significant differences in left atrial diameter between the two modalities, however, by the end of hemodiafiltration a decrease was observed $(p<0.001)$. In contrast, similar changes were not found during hemodialysis $(\mathrm{p}=0.11)$ (Figure 6.). Regarding end diastolic left ventricular diameter and the left ventricular ejection fraction no differences or changes were observed in either of the treatments. Body weight and body mass index (BMI) decreased significantly in both modalities (Table 4.). Importantly, the effective volume removals did not differ significantly between the two renal replacement modalities. During both therapies positive correlations were found between the left ventricular mass index, and the QTmax, QTmaxc, and QTd ( $<$ <0.05) (Table 5.). No malignant ventricular arrhythmias appeared, but the occurrence of ventricular premature beats was significantly higher during hemodialysis $(\mathrm{p}=0.018)$ (Table 6.). The ratio of ventricular premature beats to the total number of heart beats differed significantly (Figure 7.). There were no significant correlations between QT parameters and premature ventricular complexes, while in the case of hemodialysis QTmax and QTc correlated positively with the number of premature atrial complexes (Table 7.). The left ventricular ejection fraction correlated negatively with the number of ventricular premature beats in both treatment modalities (HDF r $=-0.55, \mathrm{p}=0.0015$, HD r=-0.36, $\mathrm{p}=0.046)$.

\section{Discussion}

The lengthening of the QT interval and dispersion may result in an increased risk of malignant ventricular arrhythmias and the danger of sudden cardiac death[11-13]. Mortality rate of patients on chronic hemodiafiltration programs is $35 \%$ lower than those receiving hemodialysis[14]. Previously, it has been shown that in the case of hemodiafiltration the clearance of small- and medium- molecular weight substances is higher compared to conventional treatment, and the concentrations of acute phase proteins, and inflammatory mediators (C-reactive protein, interleukin-1, -6, $\beta-2$ microglobulin) do not rise during such treatments or thereafter[15-18]. The suppression of inflammatory reactions may contribute to the positive changes in the frequency of anemia during hemodiafiltration. Besides the known factors, it could be also evaluated that patients receiving hemodiafiltration treatment for their 
renal disease better enjoy the benefits resulting from the reduction in arrhythmia incidences and from the slower progression of the cardiac target organ damage[19,20]. In our investigation hemodiafiltration did not cause significant changes in QT parameters, while hemodialysis significantly lengthened all the studied ECG markers. This difference suggests the beneficial effect of hemodiafiltration on the ventricular repolarizational electrocardiographic markers. This can be the consequence of various causes. On one hand the significant positive correlation between the QTmaxc and the sodium during hemodialysis directs the attention to the possible effects of the changes in electrolyte concentrations. On the other hand the increase in ventricular frequency observed during hemodialysis could be the result of the differences in the intravascular and intracardiac dynamics of the volume state between the two renal replacement therapies. Furthermore, the larger decrease in the left atrial diameter during hemodiafiltration suggests that hemodiafiltration might have a more efficient intracardiac volume decreasing potential; however there was no significant difference in the effective volume removals between the two modalities. As no significant differences were observed between the two modalities regarding serum potassium, magnesium and phosphate we conclude that the altered behavior of electrocardiographic parameters during the different renal replacement therapies do not likely to be caused by the changes in these ionic parameters. Furthermore, during hemodiafiltration, a negative correlation was seen between both the total calcium and ionized calcium levels and the QTmaxc. Moreover, in the case of hemodialysis the serum sodium and the QTmaxc showed a significant positive correlation. These results suggest that the alterations of these arrhythmia markers may be the result of the coexistence of certain ionic imbalances and renal replacement methods. Also, this phenomenon highlights the importance of the careful control of these ionic parameters and the potential benefits of electrolyte profiling over the course of the sessions in order to prevent electrocardiographic changes and to lower the risk of arrhythmogenesis. The possible reasons for the significant differences in QT parameters observed between the two modalities even at the beginning of treatments have not been clearly established and needs further investigation. The left ventricular mass index, QTmax, QTmaxc, and QTd showed a positive correlation regardless of the type of renal replacement modality, which confirms the role the myocardium's mass growth plays in arrhythmogenesis. Although malignant ventricular arrhythmias were not observed in any one of the modalities applied, ventricular premature complexes appeared significantly more often during hemodialysis. No significant correlations were found between QT parameters and premature ventricular complexes, while in the case of hemodialysis QT and QTc correlated positively with premature atrial complexes. These 
results attract the attention to the possible role of the increased atrial and ventricular intracardiac pressures and myocardial wall stress in the genesis of both atrial and ventricular arrhythmias in the case of conventional hemodialysis. A degree of similarity between the volume removals might be indicated by the kinetics in the changes of blood pressure, but the systolic and diastolic data showed significant differences even at the start of the treatments, which may affect the results. The relationship between the left ventricular systolic dysfunction and the tendency to ventricular arrhythmias has been confirmed by our results, and this was found to be valid in both renal replacement modalities.

\section{Conclusion}

During hemodiafiltration, in contrast to hemodialysis, the studied electrocardiographic markers do not change significantly. This might be the result of a more balanced but increased intracardiac and intravascular volume reductions, the more effective balancing of the sodium concentration, and the already proven powerful antioxidant and anti-inflammatory effects, positive influence on the lipid profile, the more efficient removal of the small and medium molecular-weight solutes and uremic toxins that can be observed during hemodiafiltration.

\section{Study limitation}

The number of the studied patients is relatively small, however all patients suitable for clinical investigations were enrolled from our center. The determination of long term effects of hemodiafiltration on cardiac arrhythmias was not possible during the present study, so further investigations are needed to get a clearer picture on this issue. Our results apply only to our selected group of patients with a low frequency of coronary artery disease.

\section{Competing Interests}

None of the authors have relationship with companies that may have a financial or nonfinancial interest in the information contained in the manuscript.

\section{Acknowledgements}

Our research project was sponsored by the TÁMOP-4.2.2.A-11/1/KONV-2012-0045 project. This project is implemented through the New Hungary Development Plan, co-financed by the European Union and the European Social Fund. 
References

1. Sra J, Dhala A, Blanck Z, Deshpande S, Cooley R, Akhtar M. Sudden cardiac death. Curr. Probl. Cardiol. 1999;24:461-538.

2. Panikkath R, Reinier K, Uy-Evanado A, Teodorescu C, Gunson K, Jui J, et al. Electrocardiographic predictors of sudden cardiac death in patients with left ventricular hypertrophy. Ann. Noninvasive Electrocardiol. Off. J. Int. Soc. Holter Noninvasive Electrocardiol. Inc. 2013;18:225-9.

3. Noseworthy PA, Peloso GM, Hwang SJ, Larson MG, Levy D, O’Donnell CJ, et al. QT interval and long-term mortality risk in the Framingham Heart Study. Ann. Noninvasive Electrocardiol. Off. J. Int. Soc. Holter Noninvasive Electrocardiol. Inc. 2012;17:340-8.

4. Kanbay M, Afsar B, Goldsmith D, Covic A. Sudden death in hemodialysis: an update. Blood Purif. 2010;30:135-45.

5. Sicouri S, Glass A, Ferreiro M, Antzelevitch C. Transseptal dispersion of repolarization and its role in the development of Torsade de Pointes arrhythmias. J. Cardiovasc.

Electrophysiol. 2010;21:441-7.

6. Green D, Roberts PR, New DI, Kalra PA. Sudden cardiac death in hemodialysis patients: an in-depth review. Am. J. Kidney Dis. Off. J. Natl. Kidney Found. 2011;57:921-9.

7. Franczyk-Skora B, Gluba A, Banach M, Kozlowski D, Malyszko J, Rysz J. Prevention of sudden cardiac death in patients with chronic kidney disease. BMC Nephrol. 2012;13:1622369-13-162.

8. Canaud B, Bragg-Gresham JL, Marshall MR, Desmeules S, Gillespie BW, Depner T, et al. Mortality risk for patients receiving hemodiafiltration versus hemodialysis: European results from the DOPPS. Kidney Int. 2006;69:2087-93.

9. Canaud B, Bosc JY, Leray-Moragues H, Stec F, Argiles A, Leblanc M, et al. On-line haemodiafiltration. Safety and efficacy in long-term clinical practice. Nephrol. Dial. Transplant. Off. Publ. Eur. Dial. Transpl. Assoc. - Eur. Ren. Assoc. 2000;15 Suppl 1:60-7.

10. Schmid H, Schiffl H. Hemodiafiltration and survival of end-stage renal disease patients: the long journey goes on. Int. Urol. Nephrol. 2012;

11. Elming H, Holm E, Jun L, Torp-Pedersen C, Kober L, Kircshoff M, et al. The prognostic value of the QT interval and QT interval dispersion in all-cause and cardiac mortality and morbidity in a population of Danish citizens. Eur. Heart J. 1998;19:1391-400.

12. Napolitano C, Priori SG, Schwartz PJ. Significance of QT dispersion in the long QT syndrome. Prog. Cardiovasc. Dis. 2000;42:345-50.

13. Antzelevitch C. Heterogeneity and cardiac arrhythmias: an overview. Heart Rhythm Off. J. Heart Rhythm Soc. 2007;4:964-72.

14. Locatelli F, Marcelli D, Conte F, Limido A, Malberti F, Spotti D. Comparison of mortality in ESRD patients on convective and diffusive extracorporeal treatments. The Registro Lombardo Dialisi E Trapianto. Kidney Int. 1999;55:286-93. 
15. Kerr PB, Argiles A, Flavier JL, Canaud B, Mion CM. Comparison of hemodialysis and hemodiafiltration: a long-term longitudinal study. Kidney Int. 1992;41:1035-40.

16. Canaud B, Wizemann V, Pizzarelli F, Greenwood R, Schultze G, Weber C, et al. Cellular interleukin-1 receptor antagonist production in patients receiving on-line haemodiafiltration therapy. Nephrol. Dial. Transplant. Off. Publ. Eur. Dial. Transpl. Assoc. - Eur. Ren. Assoc. 2001;16:2181-7.

17. Spalding EM, Chamney PW, Farrington K. Phosphate kinetics during hemodialysis: Evidence for biphasic regulation. Kidney Int. 2002;61:655-67.

18. Blankestijn PJ, Ledebo I, Canaud B. Hemodiafiltration: clinical evidence and remaining questions. Kidney Int. 2010;77:581-7.

19. Ok E, Asci G, Toz H, Ok ES, Kircelli F, Yilmaz M, et al. Mortality and cardiovascular events in online haemodiafiltration (OL-HDF) compared with high-flux dialysis: results from the Turkish OL-HDF Study. Nephrol. Dial. Transplant. Off. Publ. Eur. Dial. Transpl. Assoc. Eur. Ren. Assoc. 2013;28:192-202.

20. Ohtake T, Oka M, Ishioka K, Honda K, Mochida Y, Maesato K, et al. Cardiovascular protective effects of on-line hemodiafiltration: comparison with conventional hemodialysis. Ther. Apher. Dial. Off. Peer-Rev. J. Int. Soc. Apher. Jpn. Soc. Apher. Jpn. Soc. Dial. Ther. 2012;16:181-8. 


\section{Figure legend}

Figure 1. During hemodiafiltration, the maximal QT interval (QTmax) and the corrected maximal QT interval (QTmaxc) did not change significantly, however, at the $30^{\text {th }}$ minute of the hemodialysis these values became prolonged compared to the baseline levels. $\left({ }^{*} p<0.05\right)$ HD: hemodialysis, HDF: hemodiafiltration

Figure 2. During hemodialysis the QT dispersion (QTd) and the corrected QT dispersion (QTdc) are prolonged in the $30^{\text {th }}$ minute, while in the case of hemodiafiltration no changes are observed. $(* \mathrm{p}<0.05)$ HD: hemodialysis, HDF: hemodiafiltration

Figure 3. At the $15^{\text {th }}$ and $30^{\text {th }}$ minutes of the hemodialysis the sodium concentration showed a significant decrease compared to the baseline $\left({ }^{*} \mathrm{p}<0.05\right)$, however, there was no similar difference observed in the case of hemodiafiltration.

HD: hemodialysis, HDF: hemodiafiltration

Figure 4. The RR cycle length showed a significant prolongation by the $30^{\text {th }}$ minute of the haemodiafiltration, on the other hand, we observed a decrease in the $2^{\text {nd }}$ hour after hemodialysis. $(* \mathrm{p}<0.05) \mathrm{HD}$ : hemodialysis, HDF: hemodiafiltration

Figures 5/a. and 5/b. The changes in systolic and diastolic blood pressures (BP) during the different methods of renal replacement. $(*=p<0.05)$ HD: hemodialysis, HDF: hemodiafiltration

Figure 6. The left atrial diameter significantly decreased during hemodiafiltration.

HD: hemodialysis, HDF: hemodiafiltration $(*=\mathrm{p}<0.05)$

Figure 7. The ratio of ventricular premature beats (VPBs) was significantly higher in the case on conventional hemodialysis.

$(* \mathrm{p}<0.05)$ HDF: hemodiafiltration, HD hemodialysis 
Table 1. Clinical data of the studied population

\begin{tabular}{|l|c|}
\hline Gender (male/female) & $18 / 12$ \\
\hline Mean age (years) & $60.57 \pm 13.62$ \\
\hline $\begin{array}{l}\text { Duration of renal replacement therapy } \\
\text { (months) }\end{array}$ & $93.13 \pm 70.09$ \\
\hline Hypertension & $27 / 30(90 \%)$ \\
\hline Myocardial infarction & $3 / 30(10 \%)$ \\
\hline Ischemic heart disease & $3 / 30(10 \%)$ \\
\hline Hyperlipidemia & $5 / 30(16.7 \%)$ \\
\hline
\end{tabular}


Table 2. Laboratory and echocardiographic data of the studied population.

* indicate significant changes regarding echocardiographic parameters and laboratory findings compared to the baseline $(\mathrm{p}<0.05)$. LAD: left atrial diameter, LVMI: left ventricular mass index, EF: left ventricular ejection fraction, HD: hemodialysis, HDF: hemodiafiltration

\begin{tabular}{|c|c|c|c|c|}
\hline & \multicolumn{2}{|c|}{ HD } & \multicolumn{2}{|c|}{ HDF } \\
\hline & Before session & End of session & Before session & End of session \\
\hline$\left[\mathrm{Na}^{+}\right](\mathrm{mmol} / \mathrm{l})$ & $143.7 \pm 3.2$ & $142.86 \pm 3.3$ & $143.1 \pm 4.3$ & $142 \pm 3.2$ \\
\hline$\left[\mathrm{K}^{+}\right](\mathrm{mmol} / \mathrm{l})$ & $5.29 \pm 0.6$ & $3.8 \pm 0.28 *$ & $5.04 \pm 0.7$ & $3.75 \pm 0.3 *$ \\
\hline$\left[\mathrm{Mg}^{++}\right](\mathrm{mmol} / \mathrm{l})$ & $1 \pm 1.13$ & $0.85 \pm 0.06 *$ & $1 \pm 0.13$ & $0.87 \pm 0.06 *$ \\
\hline$\left[\mathrm{Ca}^{++}\right](\mathrm{mmol} / \mathrm{l})$ & $1.18 \pm 0.12$ & $1.31 \pm 0.06^{*}$ & $1.16 \pm 0.11$ & $1.31 \pm 0.07 *$ \\
\hline [Ca total] $(\mathrm{mmol} / \mathrm{l})$ & $2.15 \pm 0.2$ & $2.39 \pm 0.13^{*}$ & $2.13 \pm 0.18$ & $2.34 \pm 0.11^{*}$ \\
\hline$\left[\mathrm{PO}_{4}{ }^{2-}\right](\mathrm{mmol} / \mathrm{l})$ & $1.61 \pm 0.56$ & $0.73 \pm 0.23 *$ & $1.56 \pm 0.33$ & $0.72 \pm 0.18 *$ \\
\hline urea $(\mathrm{mmol} / \mathrm{l})$ & $22.37 \pm 7.2$ & $8.58 \pm 3.1 *$ & $20.91 \pm 5.1$ & $7.8 \pm 2.3 *$ \\
\hline kreatinine $(\mu \mathrm{mol} / \mathrm{l})$ & $766 \pm 182$ & $402 \pm 105^{*}$ & $761.7 \pm 182$ & $383 \pm 100 *$ \\
\hline$\overline{\mathrm{LAD}}(\mathrm{mm})$ & $45.1 \pm 6.5$ & $43.43 \pm 5.8$ & $45.43 \pm 5.2$ & $40.77 \pm 6 *$ \\
\hline septum (mm) & $13.67 \pm 1.87$ & $13.7 \pm 1.3$ & $13.97 \pm 2.1$ & $13.4 \pm 2.1$ \\
\hline posterior wall (mm) & $13.03 \pm 1.6$ & $13.17 \pm 1.2$ & $13.57 \pm 1.7$ & $13.27 \pm 1.7$ \\
\hline LVMI $\left(\mathrm{g} / \mathrm{m}^{2}\right)$ & $211 \pm 69$ & $193 \pm 64$ & $188 \pm 62$ & $185 \pm 65$ \\
\hline EF (\%) & $56.57 \pm 9.2$ & $56 \pm 7.7$ & $56.47 \pm 8.7$ & $54.57 \pm 6.8$ \\
\hline
\end{tabular}


Table 3. The correlating coefficients describing the relation between QT parameters and ionic markers. The investigation was independent of time (Spearman's rank analysis) $(* \mathrm{p}<0.05)$. HD: hemodialysis, HDF: hemodiafiltration, tCa: serum total calcium, iCa: serum ionized calcium

\begin{tabular}{|l|c|c|c|c|}
\hline HDF & QTmax & QTmaxc & QTd & QTdc \\
\hline $\mathrm{Na}^{+}$ & 0.13928 & 0.1497 & -0.14895 & -0.21304 \\
\hline $\mathrm{K}^{+}$ & 0.21096 & 0.28614 & -0.11274 & -0.11117 \\
\hline $\mathrm{Mg}^{2+}$ & 0.07835 & 0.02973 & 0.10157 & 0.1498 \\
\hline $\mathrm{tCa}$ & -0.27192 & $\mathbf{- 0 . 5 4 6 4 7} *$ & -0.03585 & -0.04488 \\
\hline $\mathrm{iCa}^{2+}$ & -0.30389 & $\mathbf{- 0 . 5 0 7 7 5 *}$ & -0.09232 & -0.09422 \\
\hline $\mathrm{PO}_{4}{ }^{2-}$ & 0.11576 & 0.21603 & 0.08655 & 0.12102 \\
\hline $\mathbf{H D}$ & QTmax & QTmaxc & QTd & QTdc \\
\hline $\mathrm{Na}^{+}$ & 0.36653 & $\mathbf{0 . 4 6 1 9 9} *$ & 0.13464 & 0.0578 \\
\hline $\mathrm{K}^{+}$ & 0.12411 & 0.07758 & -0.01079 & -0.16192 \\
\hline $\mathrm{Mg}^{2+}$ & 0.04189 & -0.04976 & 0.23717 & 0.09602 \\
\hline $\mathrm{tCa}^{2+}$ & -0.17572 & -0.0128 & 0.11822 & 0.11421 \\
\hline $\mathrm{iCa}^{2+}$ & 0.03974 & 0.1641 & -0.05534 & -0.02377 \\
\hline $\mathrm{PO}_{4}{ }^{2-}$ & 0.27964 & 0.11821 & 0.31424 & 0.12578 \\
\hline
\end{tabular}


Table 4. Body weight (BW) and body mass index (BMI) before and after hemodialysis (HD) and hemodiafiltration (HDF). * indicate significant changes compared to the baseline $(p<0.05)$. Volume removals between hemodialysis and hemodiafiltration did not differ significantly.

\begin{tabular}{|l|c|c||c|c|}
\hline & BW before $(\mathbf{k g})$ & BW after $(\mathbf{k g})$ & BMI before & BMI after \\
\hline HD & $67.47 \pm 14.44$ & $\mathbf{6 5 . 3} \pm \mathbf{1 4 . 7 4} *$ & $24.4 \pm 4.2$ & $\mathbf{2 3 . 6} \pm \mathbf{4 . 3} *$ \\
\hline HDF & $67.4 \pm 14.34$ & $\mathbf{6 5 . 2 9} \pm \mathbf{1 4 . 3 3} *$ & $24.38 \pm 4.12$ & $\mathbf{2 3 . 6} \pm \mathbf{4 . 1 4} *$ \\
\hline
\end{tabular}


Table 5. The relation between QT parameters and the left ventricular mass index (Spearman's rank analysis). Correlational coefficients ( $\mathrm{r}$ values) are shown $(* \mathrm{p}<0.05)$

HD: hemodialysis, HDF: hemodiafiltration

\begin{tabular}{|lcc|}
\hline QT parameter & r value HDF & r value HD \\
QTmax 0 minute & $*_{\mathbf{0 . 4 8 9 3 3}}$ & $\boldsymbol{*}_{\mathbf{0 . 6 1 1 4 7}}$ \\
QTmax 15 minute & $\boldsymbol{*}_{\mathbf{0 . 4 5 2 0 0}}$ & $\boldsymbol{*}_{\mathbf{0 . 5 0 1 6 4}}$ \\
QTmax 30 minute & $\boldsymbol{*}_{\mathbf{0 . 4 5 0 7 3}}$ & 0.35027 \\
QTmax 240 minute & $\boldsymbol{*}_{\mathbf{0 . 4 2 7 5 8}}$ & $\boldsymbol{*}_{\mathbf{0 . 4 9 3 6 8}}$ \\
QTmax after HD & $\boldsymbol{*}_{\mathbf{0 . 3 7 0 6 5}}$ & $\boldsymbol{*}_{\mathbf{0 . 5 5 2 2 4}}$ \\
QTmax c 0 minute & $\boldsymbol{*}_{\mathbf{0 . 5 2 7 8 3}}$ & $\boldsymbol{*}_{\mathbf{0 . 5 3 0 1 5}}$ \\
QTmax c 15 minute & 0.31079 & 0.40198 \\
QTmax c 30 minute & $\boldsymbol{*}_{\mathbf{0 . 4 5 6 9 2}}$ & $\boldsymbol{*}_{\mathbf{0 . 4 3 1 2 8}}$ \\
QTmax c 240 minute & $\boldsymbol{*}_{\mathbf{0 . 4 6 9 9 4}}$ & $\boldsymbol{*}_{\mathbf{0 . 3 8 9 5 5}}$ \\
QTmax c after HD & 0.30301 & $\boldsymbol{*}_{\mathbf{0 . 3 9 7 7 7}}$ \\
QTd 0 minute & 0.20413 & 0.10882 \\
QTd 15 minute & 0.17227 & 0.31938 \\
QTd 30 minute & 0.14779 & 0.32537 \\
QTd 240 minute & $*_{\mathbf{0 . 4 5 8 7 4}}$ & $\boldsymbol{*}_{\mathbf{0 . 4 0 1 4 4}}$ \\
QTd after HD & 0.18338 & -0.11899 \\
QTd c 0 minute & 0.02905 & 0.01096 \\
QTd c15 minute & -0.01944 & 0.14868 \\
QTd c 30 minute & -0.01220 & 0.27103 \\
QTd c 240 minute & 0.34950 & 0.25348 \\
QTd c after HD & 0.12099 & -0.30186 \\
\hline
\end{tabular}


Table 6. Number of premature atrial complexes (PVCs) and premature ventricular complexes (PVCs) during hemodialysis and hemodiafiltration. The occurrence of PVCs was significantly higher during hemodialysis. The number of premature atrial complexes was higher in the case of hemodialysis, but this difference did not prove to be significant. * $\mathrm{p}<0.05$

\begin{tabular}{|l|c|c|}
\hline & Number of PACs & Number of PVCs \\
\hline HD & $363.6 \pm 690$ & $\mathbf{2 5 6 . 2} \pm \mathbf{6 5} 7^{*}$ \\
\hline HDF & $350.4 \pm 765$ & $183.1 \pm 476$ \\
\hline
\end{tabular}


Table 7. Significant correlations between electrocardiographic variables and premature atrial complexes during hemodialysis ( $\mathrm{r}$ values showing correlations between the studied parameters). No such significant correlations were found during hemodiafiltration.* $\mathrm{p}<0.05$. QTmax: QT interval, QTmaxc: corrected QT interval, PACs: premature atrial complexes

\begin{tabular}{|l|l|}
\hline QTmax & PACs \\
\hline 0 min & $\mathbf{r}=\mathbf{0 . 5 3} *$ \\
\hline 15 min & $\mathbf{r}=\mathbf{0 . 4 4} *$ \\
\hline $30 \mathrm{~min}$ & $\mathbf{r}=\mathbf{0 . 4 1} *$ \\
\hline 240 min & $\mathbf{r}=\mathbf{0 . 2 7} *$ \\
\hline 2 hours after treatment & $\mathbf{r}=\mathbf{0 . 3 7} *$ \\
\hline QTmaxc & $\mathbf{P A C s}$ \\
\hline 0 min & $\mathbf{r}=\mathbf{0 . 4 6} *$ \\
\hline 15 min & $\mathbf{r}=\mathbf{0 . 4 7} *$ \\
\hline 30 min & $\mathbf{r}=\mathbf{0 . 4 6} *$ \\
\hline 240 min & $\mathbf{r}=\mathbf{0 . 3 9} *$ \\
\hline 2 hours after treatment & $\mathbf{r}=\mathbf{0 . 5 2} *$ \\
\hline
\end{tabular}

